九州大学学術情報リポジトリ

Kyushu University Institutional Repository

\title{
ON A POLYNOMIAL REPRESENTATION OF FINITE LINEAR CELLULAR AUTOMATA
}

Nohmi, Masaya

Department of Artificial Intelligence, Kyushu Institute of Technology

https://doi.org/10.5109/13414

出版情報: Bulletin of informatics and cybernetics. 24 (3/4), pp.137-145, 1991-03. Research Association of Statistical Sciences

バージョン:

権利関係 : 


\title{
ON A POLYNOMIAL REPRESENTATION OF FINITE LINEAR CELLULAR AUTOMATA
}

By

\author{
Masaya Noнmi*
}

\begin{abstract}
This paper decides the behaviour of cellular automata, including the existence of fixed points and the order of configurations, using the polynomial representation of configurations with respect to a basis derived from a series of state transition. We are mainly concerned with cellular automata with local transition rile 90 since the method described here can be used for any finite cellular automata with a little alternations [7].
\end{abstract}

\section{Introduction}

In this paper, we are concerned with cellular automata that have linearly-sited cells, and each cell of them has value 0 or 1 . S. Wolfram gave a standard numbering to transition rules of this kind of cellular automata [8]. We are mainly concerned with local transition rule 90 , that is decided so that the value of a cell is the sum of the values of its two nearest neighbourhoods at the previous time step, where the sum is computed modulo 2 . We assume the boundary condition to be null, that is, for the leftmost cell, the value of the left-hand neighbourhood is regarded as 0 , and for the rightmost cell, it is treated similarly.

Figure 1 gives an example of state transition of eight cells. The first column denotes the initial configuration. It can be selected arbitrarily. In this case, we choose the first vector of the standard basis. The second column denotes the configuration at the next time step and so on. We see the state transition to be periodic, and its order is fourteen.

\footnotetext{
* Department of Artificial Intelligence, Kyushu Institute of Technology, Iizuka 820, Japan
} 


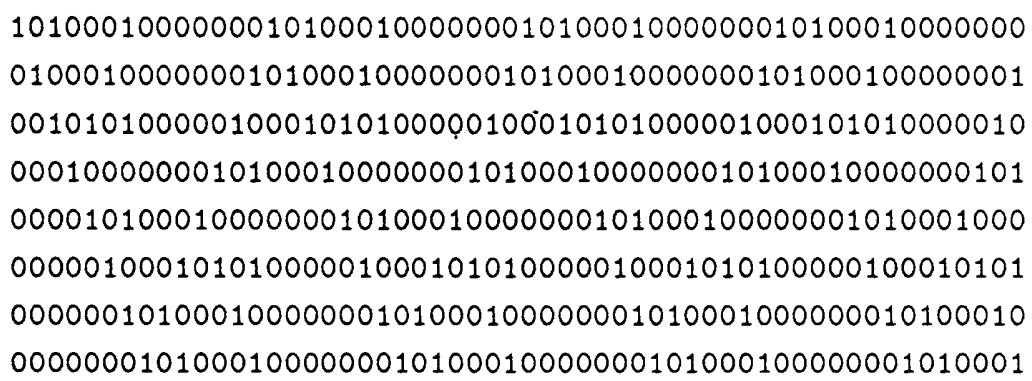

Fig. 1. An example of the state transition.

We treat the automaton in more mathematical manner. Let $F_{2}=\{0,1\}$ be the finite field of order 2, and let $V_{m}$ be the vector space $F_{2}^{m-1} . V_{m}$ is the set of all configurations of $m-1$ cells. For convenience we use $m$ instead of $m-1$. The transition rule is a linear transformation on $V_{\mathrm{m}}$ and its representative matrix $A_{m}$ of degree $m-1$ is

$$
A_{m}=\left(\begin{array}{ccccccc}
0 & 1 & & & & \\
1 & 0 & 1 & & & \\
& 1 & 0 & 1 & & \\
& & & \cdot & \ddots & \ddots & \\
& & & \ddots & \ddots & \ddots & \\
& & & & \ddots & 1 \\
& & & & 1 & 0
\end{array}\right) .
$$

For convenience, we denote it as follows:

$$
c a-90(m)=\left(V_{m}, A_{m}\right) \text {. }
$$

\section{The Polynomial Representation of Configurations}

Let $e_{1}, e_{2}, \ldots, e_{m-1}$ be the standard basis of $V_{m}$. We choose $m-1$ vectors

$$
f_{i}=A_{m}^{i-1} e_{1}(i=1, \ldots, m-1)
$$

from $V_{m}$. Since the matrix $\left(f_{1}, f_{2}, \ldots, f_{m-1}\right)$ is regular, $f_{1}, f_{2}, \ldots, f_{m-1}$ is a basis of $V_{m}$. We call it the transition basis. We defined $V_{m}$ as a vector space over $F_{2}$, but we can regard it as an $F_{2}[t]$-module by defining the polynomial multiplication of a vector $v \in$ $V_{m}$ and a polynomial $f(t) \in F_{2}[t]$ as follows:

$$
f(t) \cdot v=f\left(A_{m}\right) v .
$$

Of course, substituting another matrix for $f(t)$, we get another $F_{2}[t]$-module, and in fact, we use another one later. But if $e\left(=e_{1}\right)$ is taken as the vector $v$, we always substitute $A_{m}$ for $f(t)$. Since the vectors $f_{1}, f_{2}, \ldots, f_{m-1}$ are a basis of $V_{m}$, any vector $v$ $\in F_{2}$ can be uniquely represented as follows: 


$$
v=a_{1} f_{1}+a_{2} f_{2}+\ldots+a_{m-1} f_{m-1}\left(a_{i} \in F_{2}\right) .
$$

By the definition of the polynomial multiplication, we can rewrite the above equation as

$$
v=\left(a_{1}+a_{2} t+\ldots+a_{m-1} t^{m-2}\right) \cdot e_{1} .
$$

Therefore we can uniquely choose a polynomial $f(t) \in F_{2}[t]$ satisfying the equation $v=$ $f(t) \cdot f_{1}$ under the condition $\operatorname{deg}(f(t)) \leq m-2$. We call the polynomial $f(t)$ the polynomial representation of $v$, and emphasizing the configuration $v$, we often denote it by $f_{v}(t)$.

We define another square matrix $B_{m}$ of degree $m-1$ by

$$
B_{m}=\left(\begin{array}{cccccc}
0 & & & & & \\
1 & 0 & & & \\
& 1 & 0 & & & \\
& & \ddots & \ddots & & \\
& & & \ddots & 0 & \\
& & & & 0 & 0
\end{array}\right) .
$$

Similarly to the case of $A_{m}$, we get $F_{2}[t]$-module by defining the polynomial multiplication on $V_{m}$ as follows:

$$
f(t) \cdot v=f\left(B_{m}\right) v .
$$

If $f\left(=f_{1}\right)$ is taken as the vector $v$, we always use the matrix $B_{m}$. If a polynomial $f(t) \in$ $F_{2}[t]$ is written as

$$
f(t)=a_{1}+a_{2} t+\ldots+a_{m-1} t^{m-2}\left(a_{i} \in F_{2}\right),
$$

we have

$$
f(t) \cdot f={ }^{t}\left(a_{1}, a_{2}, \ldots, a_{m-1}\right) .
$$

\section{The Polynomial $\varphi_{\mathbf{m}}(t)$}

In describing the behavior of $c a-90(m)$, we often use the polynomial $\varphi_{m}(t)$. We recall the definition of it and some formulas from Matsumoto [6]. The polynomial $\varphi_{m}(t) \in F_{2}[t]$ is defined by the equations $\varphi_{0}(t)=0, \varphi_{1}(t)=1$ and

$$
\varphi_{m}(t)=t \varphi_{m-1}(t)+\varphi_{m-2}(t)(m \geq 2) .
$$

From the definition, we have some fundamental formulas. For any $m \geq 1$,

$$
\begin{aligned}
& \operatorname{deg}\left(\varphi_{m}(t)\right)=m-1 \\
& \left(\begin{array}{ll}
0 & 1 \\
1 & t
\end{array}\right)^{m}=\left(\begin{array}{rr}
\varphi_{m-1}(t) & \varphi_{m}(t) \\
\varphi_{m}(t) & \varphi_{m+1}(t)
\end{array}\right),
\end{aligned}
$$




$$
\varphi_{m+1}(t) \varphi_{m-1}(t)+\varphi_{m}(t)^{2}=1 .
$$

Next, we see the factorization of $\varphi_{m}(t)$ in $\bar{F}_{2}$, where $\bar{F}_{2}$ is the algebraic closure of $F_{2}$. Now, we set

$$
\begin{aligned}
& m=2^{s} m^{\prime}\left(\left(m^{\prime}, 2\right)=1\right), \\
& m_{1}=\frac{m^{\prime}-1}{2}
\end{aligned}
$$

and we denote the primitive $m^{\prime}$-th root of unity by $\zeta$, and set $\eta_{i}=\zeta^{i}+\zeta^{-i}$. We have the factorization of $\varphi_{m}(t)$ as follows:

$$
\varphi_{m}(t)=t^{2^{s}-1} \prod_{i=1}^{\mathrm{m}_{1}}\left(t-\eta_{i}\right)^{2^{s}+1}
$$

\section{Lemmas}

In this section, we give a few lemmas, and decide the Jordan normal form of $A_{m}$.

Proposition 4.1. If $m \geq 2$ and $e \in V_{m}\left(=F_{2}^{m-1}\right)$, then

$$
\phi_{m}(t) e=0 .
$$

Proof. The proof is by induction on $m$. For $m=2,3$, it is trivial. Now, assume the theorem to be true in the case of $m-2 ; \varphi_{m-2}(t) e=0$ on $V_{m-2}$. Correcting errors derived from the difference between $V_{m-2}$ and $V_{m}$, we have $\varphi_{m-2}(t) e=e_{m-2}$ on $V_{m}$. Similarly, $\varphi_{m-1}(t) e=e_{m-1}$ holds on $V_{m}$. Therefore, we have

$$
\varphi_{m}(t) e=\left(t \varphi_{m-1}(t)+\varphi_{m-2}(t)\right) e=A_{m} e_{m-1}+e_{m-2}=0,
$$

as required.

PROPOSITION 4.2. If $\varphi_{m}(t)$ is written as

$$
\varphi_{m}(t)=t^{m-1}+a_{m-2} t^{m-2}+\ldots+a_{1} t+a_{0},
$$

then

$$
A_{m} f_{m-1}=\sum_{i=1}^{m-1} a_{i-1} f_{i} .
$$

Proof. From Proposition 4.1, we have

$$
\begin{aligned}
0 & =\varphi_{m}(t) e \\
& =\left(A_{m}^{m-1}+a_{m}-2 A_{m}^{m-2}+\ldots+a_{1} A_{m}+a_{0}\right) e \\
& =A_{m}^{m-1} e+a_{m-2} f_{m-1}+\ldots+a_{1} f_{2}+a_{0} f_{1} \\
& =A_{m} f_{m-1}+\sum_{i=1}^{m-1} a_{i-1} f_{i} .
\end{aligned}
$$

Transposing the term $A_{m} f_{m-1}$ to the left-hand side, we get the result.

Let ${ }^{t} \widetilde{A}_{m}$ be the representative matrix of the transition rule of a $c a-90(\mathrm{~m})$ with respect to the basis $f_{1}, f_{2}, \ldots, f_{m-1}$. It is transposed for convenience. The index $m$ is 
often omitted. By the Proposition 4.2, setting

$$
\varphi_{m}(t)=t^{m-1}+a_{m-2} t^{m-2}+\ldots+a_{1}+a_{0},
$$

we have,

$$
\widetilde{\mathrm{A}}=\left(\begin{array}{cccccc}
0 & 1 & & & & \\
& 0 & 1 & & & \\
& & 0 & 1 & & \\
& & & \ddots & \ddots & \\
& & & & \ddots & \\
& & & & 0 & 1 \\
a_{0} & a_{1} & & \ldots & a_{m-3} & a_{m-2}
\end{array}\right)
$$

It is easy to confirm the characteristic polynomial of the matrix (6) is $\varphi_{m}(t)$. Let $H_{m^{\prime}}$ be the set of non-zero roots of the polynomial $\varphi_{m}(t)$. From factorization (5), we have $H_{m^{\prime}}$ $=\left\{\eta_{i}=\zeta^{i}+\xi^{-i} \mid i=1, \ldots, m_{1}\right\}$. Denoting the $n \times n$ Jordan block corresponding to the eigenvalue $\alpha \in \bar{F}_{2}$ by $J_{n}(\alpha)$, we have the Jordan normal form $J$ of $\widetilde{A}$ as follows:

$$
J=\bigoplus_{i=1}^{m_{1}} J_{2^{s+1}}\left(\eta_{i}\right) \oplus J_{2^{s-1}}(0) .
$$

Next we give the matrix $P$ satisfying the equation

$$
\widetilde{A}=P J P^{-1} \text {. }
$$

For $\alpha \in \overline{F_{2}}$, we define an $(m-1) \times n$ matrix $P_{n}(\alpha)$ by

$$
P_{n}(\alpha)=\left(\begin{array}{ccccc}
1 & & & & \\
\alpha & 1 & & & \\
\alpha^{2} & \left(\begin{array}{c}
2 \\
1
\end{array}\right) \alpha & 1 & & \\
\vdots & \vdots & \vdots & \ddots & 1 \\
\vdots & \vdots & \vdots & & \vdots \\
\alpha^{m-2} & \left(\begin{array}{c}
m-2 \\
1
\end{array}\right) \alpha^{m-3} & \left(\begin{array}{c}
m-2 \\
2
\end{array}\right) \alpha^{m-4} & \ldots & \left(\begin{array}{c}
m-2 \\
n-1
\end{array}\right) \alpha^{m-n-1}
\end{array}\right),
$$

that is, the $(i, j)$-element of $P_{n}(\alpha)$ is

$$
\left(\begin{array}{c}
i-1 \\
j-1
\end{array}\right) \alpha^{i-j}(1 \leq i \leq m-1,1 \leq j \leq n) .
$$

The matrix $P$ satisfying the equation (8) is as follows:

$$
P=\left(P_{2^{s+1}}\left(\eta_{1}\right), \ldots, P_{2^{s+1}}\left(\eta_{m_{1}}\right), P_{2^{s-1}}(0)\right) .
$$


Proposition 4.3. If a polynomial $f(t) \in F_{2}[t]$ satisfies $\operatorname{deg}(f(t)) \leq m-2$, then for any $\alpha \in F_{2}$,

$$
{ }^{t} P_{n}(\alpha) f(t) \cdot f_{1}=\left(\begin{array}{c}
f(\alpha) \\
f^{\prime}(\alpha) \\
\frac{1}{2} f^{\prime \prime}(\alpha) \\
\vdots \\
\frac{1}{(n-1) !} f^{(n-1)}(\alpha)
\end{array}\right) .
$$

\section{Applications to Transition Diagrams}

Vector space $V_{m}$ is a direct sum of the set of infinite images and the set of infinite kernels; $V_{m}=\operatorname{Im} A^{\infty} \oplus \operatorname{Ker} A^{\infty}$. First, we decide the construction of the kernel tree that is the transition diagram whose verteces are restricted to the infinite kernel and the edge from 0 to 0 is removed. It is a binary tree, except the root has only one edge, and each leaf has the same depth. So, we can decide the construction of the kernel tree only by the depth of it. A vertex corresponding to infinite image appears as a node on a loop, and each node is connected to a tree isomorphic to the kernel tree. So, we can decide the global constitution of transition diagram by the order of each loop.

THEOREM 5.1. We can select bases of $\operatorname{Im} A^{\infty}$ and $\operatorname{Ker} A^{\infty}$ as follows:

$$
\begin{aligned}
& \operatorname{Im} A^{\infty}=\left\langle t^{2^{s}}, t^{2^{s}+1}, \ldots, t^{m-1}\right\rangle_{F_{2}}, \\
& \operatorname{Ker} A^{\infty}=\left\langle\frac{\varphi_{m}(t)}{t}, \frac{\varphi_{m}(t)}{t^{2}}, \ldots, \frac{\varphi_{m}(t)}{t^{2^{s}-1}}\right\rangle_{F_{2}} .
\end{aligned}
$$

For any configuration, we can decide whether it is an element of $\operatorname{Im} A^{\infty}$ or not, by the next theorem.

THEOREM 5.2. For any configuration $x \in V_{m}$,

$$
x \in \operatorname{Im} A^{\infty} \Leftrightarrow f_{x}(t) \equiv 0\left(\bmod \operatorname{deg} 2^{s}\right) .
$$

Next, we decide the order of each configuration, using the polynomial representation. Let $A$ be the set of all orders. It is easily seen that $\Lambda=\left\{1,2, \ldots, 2^{s+1}\right\} \times\left\{\right.$ ord $\left.\left(\eta_{i}\right)\right\} \cup$ $\{1\}$. For each $\lambda=2^{i} \lambda^{\prime} \in \Lambda\left(\lambda^{\prime}:\right.$ odd $)$, we define the discriminant as follows:

$$
\Phi_{\lambda}(t)=\prod_{\operatorname{ord}\left(\eta_{\mathrm{i}}\right) \mid \lambda^{\prime}} t^{2^{s}-1}\left(t-\eta_{i}\right)^{2^{i}} .
$$

We often use the quotient polynomial $\psi_{\lambda}(t)$ of $\Phi_{\lambda}(t)$ defined as follows in stead of $\Phi_{\lambda}(t)$ :

$$
\varphi(m)=\psi_{\lambda}(t) \Phi_{\lambda}(t) .
$$

Figure 2 gives an example of the quotient polynomials in the case of $m=60$.

Theorem 5.3. For any infinite image $x$, 


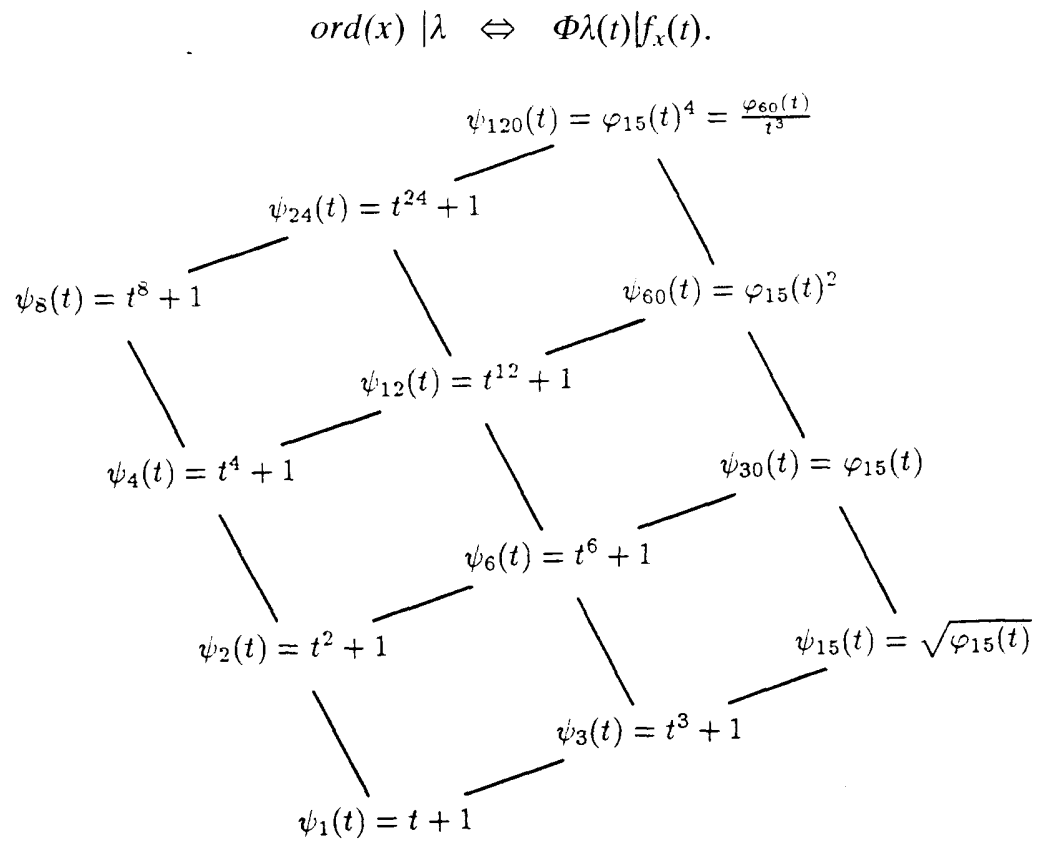

Fig. 2. An example of quotient polynomials.

\section{Local Properties of $\boldsymbol{c a}-90(\mathrm{~m})$}

If the configuration $e_{1}+e_{m-1} \in V_{m}$ is included in $\operatorname{Im} A^{\infty}$, we denote the order of it by $K(m)$. First, we discuss the existence of $K(m)$.

THEOREM 6.1. $K(m)$ exists if and only if $4 / 1 \mathrm{~m}$.

Proof. In the case $m$ is odd, the linear transformation $A$ is regular, so the result is trivial. In the case $m$ is even, we apply Proposition 5.2 to the polynomial $1+\varphi_{m-1}(t)$ which is the polynomial representation of $e_{1}+e_{m-1}$. If $m=2 m^{\prime}\left(m^{\prime}:\right.$ odd $)$, we have

$$
\varphi_{m}(t)+1 \equiv 0(\bmod \operatorname{deg} 2),
$$

so $e_{1}+e_{m-1} \in \operatorname{Im} A \infty$. If $m=2^{s} m^{\prime}\left(s \geq 2, m^{\prime}\right.$ : odd $)$, then

$$
\begin{aligned}
& \varphi_{m}(t) \equiv t^{2^{s}-1}\left(\bmod \operatorname{deg} 2^{s}\right) \\
& \varphi_{m-2}(t) \equiv t(\bmod \operatorname{deg} 2)
\end{aligned}
$$

so,

$$
\varphi_{m-1}(t)^{2}+1 \equiv t^{2^{s}}\left(\bmod \operatorname{deg} 2^{s}+2\right)
$$

Hence,

$$
\varphi_{m-1}(t)+1 \not \equiv 0\left(\bmod \operatorname{deg} 2^{s}\right) .
$$


Therefore, $K(m)$ doesn't exist in this case.

Proposition 6.2. For all $\eta_{i} \in H_{m}{ }^{\prime}$,

$$
\begin{aligned}
& \varphi_{m}\left(\eta_{i}\right)=0, \\
& \varphi_{m-1}\left(\eta_{i}\right)=1,
\end{aligned}
$$

and if $m$ is odd, then

$$
\varphi_{m-1}\left(\eta_{i}\right)^{\prime}=\eta_{i}^{-1}
$$

Proof. (12) is trivial. (13) is easily seen by substituting $\eta_{i}$ for (4). From the differentiation of (2), we have

$$
\varphi_{m}(t)=\varphi_{m-1}(t)+t \varphi_{m-1}(t)^{\prime}+\varphi_{m-2}(t)^{\prime} .
$$

Substituting $\eta_{i}$ for it, we have (14).

Proposition 6.3. If $\eta_{i} \in H_{m^{\prime}}$, then

$$
\begin{aligned}
& { }^{t} J\left(\eta_{i}\right)^{n}=\left(\begin{array}{cccc}
\eta_{i}^{n} & & & \\
\left(\begin{array}{c}
n \\
1
\end{array}\right) \eta_{i}^{n-1} & \eta_{i}^{n} & & \\
\vdots & \vdots & \ddots & \\
\left(_{m-2}^{n}\right) \eta_{i}^{n-m+2} & \left(\begin{array}{c}
n-3 \\
{ }_{m}
\end{array}\right) \eta_{i}^{n-m+3} & \ldots & \eta_{i}^{n}
\end{array}\right) \text {, } \\
& { }^{t} J_{2}\left(\eta_{i}\right)^{2^{\text {subord } m(2)-1}}=\left(\begin{array}{cc}
1 & \\
\eta_{i}^{-1} & 1
\end{array}\right) \text {. }
\end{aligned}
$$

Proof. The proof is by induction on $n$. (16) directly follows from (15).

THEOREM 6.4. If $m$ is odd, then

$$
K(m) \mid 2^{\text {subord }_{m}(2)}-1 .
$$

Proof. By the matrix ${ }^{t} P$, the configuration vector $\left(\varphi_{m-1}(t)+1\right) \cdot f$ is mapped on the vector

$$
{ }^{t} P\left(\varphi_{m-1}(t)+1\right) \cdot f=\left(\begin{array}{c}
\varphi_{m-1}\left(\eta_{1}\right)+1 \\
\varphi_{m-1}\left(\eta_{1}\right)^{\prime} \\
\varphi_{m-1}\left(\eta_{2}\right)+1 \\
\varphi_{m-1}\left(\eta_{2}\right)^{\prime} \\
\vdots \\
\varphi_{m-1}\left(\eta_{m_{1}}\right)+1 \\
\varphi_{m-1}\left(\eta_{m_{1}}\right)^{\prime}
\end{array}\right)=\left(\begin{array}{c}
0 \\
\eta_{1}^{-1} \\
0 \\
\eta_{2}^{-1} \\
\vdots \\
0 \\
\eta_{m_{1}}^{-1}
\end{array}\right)
$$

Denoting this vector by $x$, we have 


$$
\begin{aligned}
& { }^{t} J^{2^{\text {suburd } m(2)}-1} x
\end{aligned}
$$

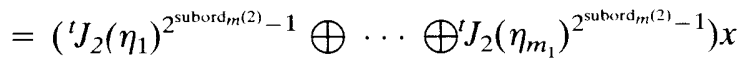

$$
\begin{aligned}
& =x \text {, }
\end{aligned}
$$

\section{by Proposition 6.3 .}

At last, we discuss the existence of fixed points.

THEOREM 6.5. There exist non-trivial fixed points if and only if $3 \mid \mathrm{m}$. In this case, the subspace of fixed points is given as follows:

$$
\left\langle\frac{\varphi_{m}(t)}{t-1}\right\rangle_{F_{2}}
$$

Proof. First, we search fixed points in the algebraic closure ${\overline{F_{2}}}^{m-1}$, and then we confirm they are included in $F_{2}^{m-1}$. Assume that $J$ has a non-trivial fixed point in ${\overline{F_{2}}}^{m-1}$, that is, there exists one Jordan block of $J$ such that

$$
{ }^{t} J_{2^{s+1}}\left(\eta_{i}\right)-I_{2^{s+1}}
$$

is degenerate. It is equivalent to $\eta_{i}=1$ and $\xi^{2 i}+\zeta^{i}+1=0$. It means $\zeta^{i}$ is a third root of unity, so we have $3 \mid \mathrm{m}$. It is easily seen that the dimension of the kernel of (17) is 1 . By

$$
P_{2^{s+1}}\left(\eta_{i}\right) \frac{\varphi_{t}(t)}{t-1}=\left\{\begin{array}{cc}
0 & \left(\eta_{i} \neq 1\right) \\
{ }^{t}(0, \ldots, 0,1) & \left(\eta_{i}=1\right)
\end{array}\right.
$$

we see the polynomial representation

$$
\frac{\varphi_{m}(t)}{t-1}
$$

gives a unique non-trivial fixed point.

\section{References}

[1] GuAn, P., He, Y.: Exact results for deterministic cellular automata with additive rules, J. of Statist. Phys, 43, 3/4 (1986).

[2] Jen, E.: Cylindrical cellular automata, Commun. Math. Phys. 118 (1988), 569-590.

[3] JEN, E.: Global properties of cellular automata, J. of Statist. Phys, 43, 1/2 (1986).

[4] LI, W.: Power spectra of regular languages and cellular automata, Complex Systems, 1 (1987), 107130.

[5] Martin, O., Odlyzko M., Wolfram, S.: Algebraic properties of cellular automata, Commun. Math. Phys, 93 (1984), 219-258.

[6] Matsumoto, M.: On cellular automaton CA - 90(m), Note, (1988).

[7] Noнмі, M.: On transition diagrams of finite cellular automata, in preparation.

[8] Wolfram, S.: Statistical mechanics of cellular automata, Rev. Mod. Phys. 55 (1982).

Received September 7, 1990

Communicated by Y. Kawahara 